\title{
Circumferential degree of carotid calcification is associated with new ischemic brain lesions after carotid artery stenting
}

\author{
Peng Lv ${ }^{1 \#}$, Aihua Ji ${ }^{2 \#}$, Ranying Zhang ${ }^{1}$, Daqiao Guo ${ }^{3}$, Xiao Tang ${ }^{3}$, Jiang Lin $^{1}$ \\ ${ }^{1}$ Department of Radiology, Zhongshan Hospital, Fudan University and Shanghai Institute of Medical Imaging, Shanghai, China; ${ }^{2}$ Department of \\ Radiology, Beijing Jishuitan Hospital, Beijing, China; ${ }^{3}$ Department of Vascular Surgery, Zhongshan Hospital, Fudan University, Shanghai, China
}

\#These authors contributed equally to this work.

Correspondence to: Jiang Lin. Department of Radiology, Zhongshan Hospital, Fudan University and Shanghai Institute of Medical Imaging, Fenglin Road 180, Shanghai 200032, China. Email: lin.jiang@zs-hospital.sh.cn.

Background: The relationship between plaque calcification and new ischemic brain lesions after carotid artery stenting (CAS) remains controversial. The purpose of this study was to determine if the circumferential degree of carotid calcification is associated with new ischemic brain lesions on diffusionweighted imaging (DWI) after CAS.

Methods: A total of 96 patients with carotid stenosis of $\geq 50 \%$ who underwent CAS were enrolled in the study. All patients underwent preoperative carotid computed tomography (CT), and preoperative and postoperative brain MRI. The brain MRI sequences included T1WI, T2WI, T2-fluid-attenuated inversion recovery (FLAIR), and DWI. The location, circumferential degree, volume, percentage volume, maximum density, mean density, Agatston score of carotid calcification, and total plaque volume were assessed and compared between patients with and without new ischemic brain lesions after CAS. Univariate and multivariate analyses were performed to evaluate predictors of new ischemic brain lesions.

Results: All of the 96 patients $(67.8 \pm 6.8$ years of age, $83.3 \%$ men) were included in the analysis. New ischemic brain lesions on DWI were observed in 40 patients (41.7\%). Patients with new ischemic brain lesions after CAS had a larger circumferential degree of calcification than those without new ischemic brain lesions $(\mathrm{P}<0.001)$. There was only a possible trend toward significance for the percentage volume of calcification between the two groups with and without new brain ischemic lesions $(\mathrm{P}=0.07)$. No significant differences were found regarding the location $(\mathrm{P}=0.18)$, volume $(\mathrm{P}=0.37)$, maximum density $(\mathrm{P}=0.44)$, mean density $(\mathrm{P}=0.39)$, Agatston score $(\mathrm{P}=0.28)$, and total plaque volume $(\mathrm{P}=0.33)$ of carotid calcification between the DWI+ and DWI- groups. In the multivariate analysis, an increased risk of new ischemic brain lesions was observed in patients with a high score for the circumferential degree of calcification [score 3; odds ratio (OR): 10.7, $\mathrm{P}<0.001$; score 4, OR: 11.7, $\mathrm{P}=0.038]$.

Conclusions: The circumferential degree of carotid calcification was associated with new ischemic brain lesions after CAS. CAS should be avoided if possible for carotid stenosis with large circumferential calcified plaques.

Keywords: Carotid artery; calcification; carotid artery stenting (CAS); ischemia

Submitted Nov 07, 2020. Accepted for publication Feb 18, 2021.

doi: 10.21037/qims-20-1244

View this article at: http://dx.doi.org/10.21037/qims-20-1244 


\section{Introduction}

Carotid artery stenting (CAS) is an established treatment approach for carotid stenosis in selected patients and has similar long-term efficacy as carotid endarterectomy. However, the most common complication of CAS is new ischemic brain lesions, which has become a topic of increasing interest in recent years $(1,2)$. The incidence of new ischemic brain lesions detected on diffusion-weighted imaging (DWI) after CAS can be as high as 50\% (3). Although most patients with new ischemic brain lesions on DWI are shown to be asymptomatic, they are more likely to develop symptomatic stroke than those without new ischemic brain lesions (4). Furthermore, it has been suggested that ischemic brain lesions can increase the risk of dementia, cognitive impairment, and mortality (5).

Carotid plaque vulnerability was found to be associated with increased susceptibility to perioperative brain DWI lesions in CAS patients (3). Plaques with components such as a large lipid-rich necrotic core, thin fibrous cap, and intraplaque hemorrhage tend to be vulnerable (6). As a common component of carotid plaques, calcification has an important impact on the pathophysiological progression of atherosclerosis $(7,8)$. However, the relationship between carotid calcification and perioperative ischemic brain lesions is still controversial. Although heavy calcification is traditionally considered to increase the CAS-related risk of stroke, much evidence has suggested that the size of the calcification is not associated with cerebral ischemia after CAS (9-11).

Computed tomography (CT) imaging provides detailed, rapid, high-resolution imaging of the carotid artery lumen and the plaque (12). It is extremely sensitive in the detection of carotid calcification. In clinical practice, we noticed that patients with diffuse calcification around the carotid circumference as shown on CT were more vulnerable to new infarction shortly after CAS. Therefore, the purpose of this study was to prospectively determine the relationship between the circumferential degree of calcification, along with other calcification characteristics, and ipsilateral new ischemic brain lesions after CAS.

\section{Methods}

\section{Patients}

Between January 2018 and September 2019, 96 patients who were referred to CAS treatment were enrolled in this study. All patients had symptomatic carotid stenosis of $\geq 50 \%$ or asymptomatic carotid artery stenosis of $\geq 70 \%$ according to the North American Symptomatic Carotid Endarterectomy Trial (NASCET) criteria (13). All patients underwent carotid CT angiography (CTA) within 1 week before CAS. Brain magnetic resonance imaging (MRI) was performed the same day as carotid CTA, and then repeated 1-3 days after CAS. The exclusion criteria included the following: prior carotid artery procedures (carotid endarterectomy or CAS), carotid occlusion, nonatherosclerotic carotid stenosis, and contraindications to contrast-enhanced CT or MRI. Vascular risk factors including hypertension, smoking, diabetes, coronary heart disease, and hyperlipidemia were also recorded. This study was approved by the ethics committee of our hospital (No. B2020-400R). Informed consent was waived due to the retrospective analysis design of the study.

\section{Carotid CTA}

All CT examinations were performed on a 320-row CT system (Aquilion ONE; Canon Medical Systems Corporation, Otawara, Japan). Scans were collimated at $0.6 \mathrm{~mm}$, with a peak kilovoltage of 120 and auto milliampere, and a rotation time of 0.5 seconds. The scan range was from the aortic arch to the skull vertex. Through use of a power injector, $50-70 \mathrm{~mL}$ (according to the patient's body weight) of contrast media (iopamidol, Iopamiron 370; Bracco, Milan, Italy) was injected into the antecubital vein at a flow rate of $5 \mathrm{~mL} / \mathrm{s}$ followed by a $25 \mathrm{~mL}$ saline flush. A bolustracking technique was used in CTA scans with a threshold of 100 Hounsfield unit (HU) measured in the aortic arch.

\section{Carotid calcification assessment}

The raw CTA images were transferred to a workstation (Vitrea fx 6.0; Vital Images, MN, USA), and multiplanar reformats were created for diagnostic reading. Assessment of carotid calcification was performed through consensus reading by two radiologists (AJ and RZ with 5 years' and 3 years' experience in neurovascular imaging, respectively) who were blinded to patients' clinical information and treatment options. Calcifications were defined as hyperdense regions with a density of $>130 \mathrm{HU}$ on CTA images (14).

Carotid calcification was evaluated on cross-sectional multiplanar reconstruction (MPR) images which were perpendicular to the longitudinal axis of the proximal internal carotid artery (ICA) and showed the maximal stenosis. Carotid calcifications were categorized as 

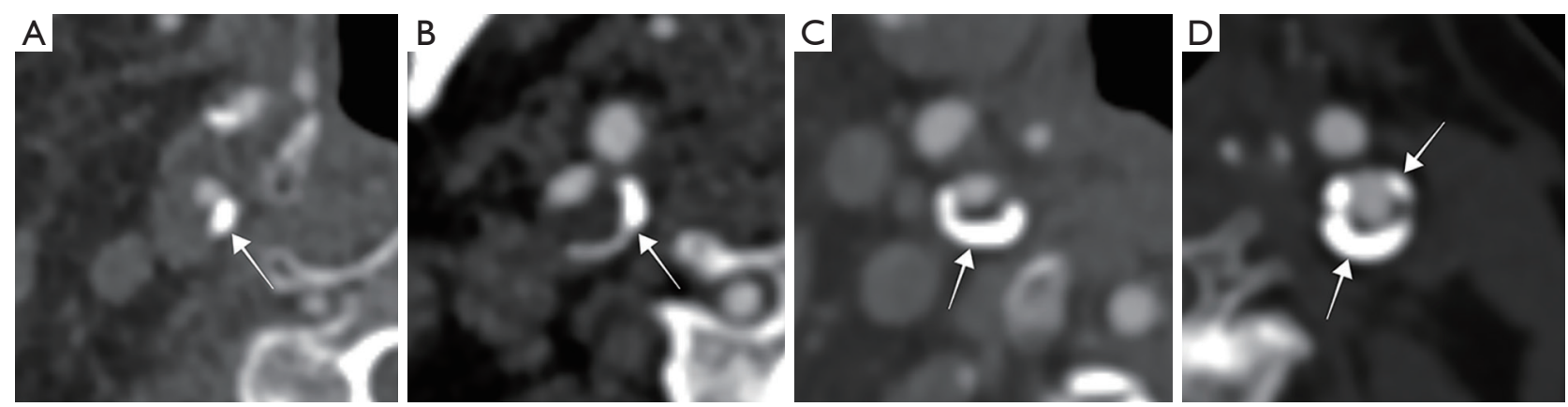

Figure 1 Examples of carotid calcification of different circumferential degrees (arrows). (A) $<90^{\circ}$ of artery circumference, (B) $90-180^{\circ}$ of artery circumference, (C) $180-270^{\circ}$ of artery circumference, and (D) $>270^{\circ}$ of artery circumference.

superficial and deep calcifications according to their locations. Superficial calcification was defined as calcification located in contact with the lumen. Deep calcification was defined as calcification located within the plaque and away from the lumen. The circumferential degree of calcification was assessed according to our own experience and previous literature $(7,15)$, and was scored on a 5 -point grading scale: 0 , none; $1,<90^{\circ}$ of artery circumference; $2,90-180^{\circ}$ of artery circumference; $3,180-270^{\circ}$ of artery circumference; and $4,>270^{\circ}$ of artery circumference (Figure 1 ). If the calcifications were distributed in different quadrants, we calculated the sum of the degrees. The volume, percentage volume, maximum density, mean density, Agatston score of carotid calcification, and total plaque volume were measured automatically or semiautomatically using plaque analysis software on the CT workstation. Percentage volume of carotid calcification was calculated as the ratio of the volume of the calcification to the total plaque volume multiplied by 100. Stenosis was also measured at the narrowest portion according to the NASCET criteria.

\section{CAS}

All treatments were performed by an experienced vascular surgeon with 10 years' experience in neurovascular intervention. Clopidogrel $(75 \mathrm{mg} / \mathrm{d})$ and aspirin $(100 \mathrm{mg} / \mathrm{d})$ were given to the patients 1 week before the procedure. CAS was carried out with the patient under local anesthesia and was performed with full systemic heparinization. A guiding catheter was placed in the ipsilateral common carotid artery. When distal embolic protection devices were used, predilatation was performed with a balloon catheter, and a stent of appropriate size was selected and deployed. If stent expansion was insufficient, postdilatation was performed.
Emboshield (Abbott Vascular, Abbott Park, IL, USA) and Filterwire (Boston Scientific, Natick, MA, USA) embolic protection devices were used in all the patients. The three types of stents used were Acculink (Abbott Vascular,), Precise (Johnson \& Johnson, Cordis, MN, USA), and Wallstent (Boston Scientific). All three carotid stents used in this study were MR safe at 3.0 T.

\section{Brain MRI}

Brain MRI was performed on a 3.0T MR scanner (Magnetom Verio; Siemens, Erlangen, Germany) with a standard head coil. Sequences of brain MRI were as follows: T1WI [repetition time (TR)/echo time (TE): 160/3.05 ms], T2WI (TR/TE: 6,000/100 ms), T2-fluid-attenuated inversion recovery (FLAIR) (TR/TE: 9,000/94.0 ms), and DWI (TR/TE: $6,400 / 86.0 \mathrm{~ms}$, b value 0 , and $1,000 \mathrm{~s} / \mathrm{mm}^{2}$ ). Slice thickness was $5 \mathrm{~mm}$ and slice spacing was $1.5 \mathrm{~mm}$ for all the sequences. New ischemic brain lesions were defined as hyperintense lesions on postoperative brain DWI that were not present on preoperative brain DWI.

\section{Statistical analysis}

Continuous variables are expressed as means \pm standard deviation and were compared using the independent $t$-test or Mann-Whitney $\mathrm{U}$ test. Categorical variables were compared with the chi-square test. Univariate and multivariate logistic regression analyses were applied to determine the association between carotid calcification characteristics and new ischemic brain lesions after CAS. Statistical significance was defined as a $\mathrm{P}$ value $<0.05$. All statistical analyses were performed using SPSS 21.0 (IBM Corp., Armonk, NY, USA). 
Table 1 Baseline characteristics of patients who underwent CAS

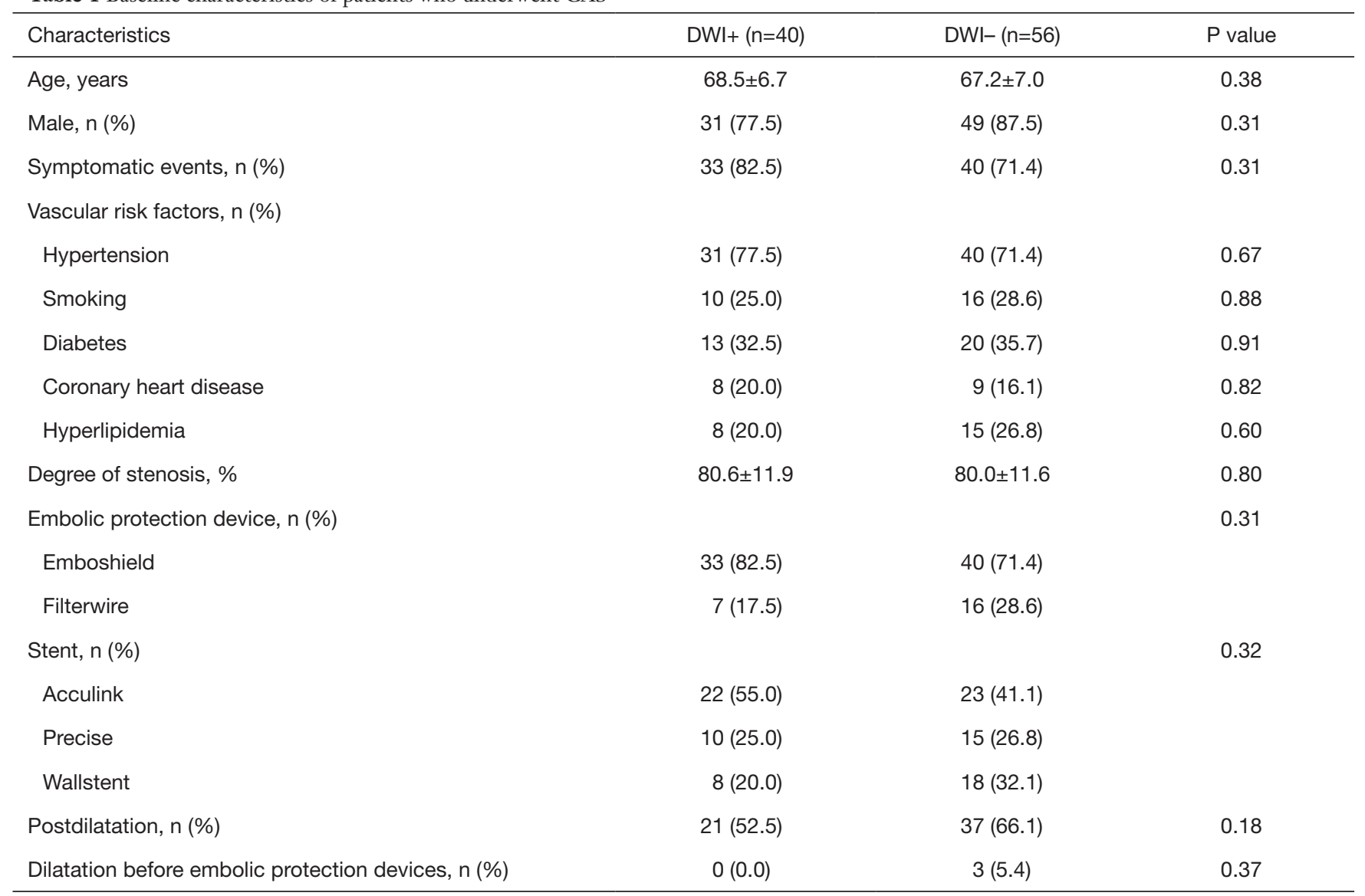

CAS, carotid artery stenting; DWI, diffusion-weighted imaging.

\section{Results}

All 96 patients were included in the analysis. Baseline characteristics of the study population are listed in Table 1 . The mean age of the 96 patients was $67.8 \pm 6.8$ years, and most patients were men $(n=80,83.3 \%)$. The baseline data were similar between the DWI+ and DWI- groups.

CAS was achieved successfully in all the 96 patients. New ischemic brain lesions on DWI were observed in 40 patients (41.7\%). Stroke symptoms occurred in 4 of the 40 patients in the DWI+ group. Compared to the DWI- group, the DWI+ group had a larger circumferential degree of carotid calcification $(\mathrm{P}=0.01)$. Furthermore, 21 of the 27 patients $(77.8 \%)$ with $>180^{\circ}$ circumference carotid calcification were detected as positive for DWI lesions (Figure 2). There was a possible trend toward significance for the percentage volume of calcification between the DWI+ and DWIgroups $(\mathrm{P}=0.07)$. No statistically significant differences were found between the DWI+ and DWI- groups with regard to all the other calcification characteristics, including the location ( $\mathrm{P}=0.18)$, volume $(\mathrm{P}=0.37)$, maximum density $(\mathrm{P}=0.44)$, mean density $(\mathrm{P}=0.39)$, Agatston score $(\mathrm{P}=0.28)$, and total plaque volume $(\mathrm{P}=0.33)$ (Table 2).

In the univariate analysis, a high score for the circumferential degree of calcification was associated with new ischemic brain lesions on DWI after CAS [score 3, odds ratio (OR): 9.7, $\mathrm{P}=0.001$; score 4, $\mathrm{OR}$ : 5.5, $\mathrm{P}=0.046$ ] (Table 3). In the multivariate analysis, this association remained and became more significant after adjustments for age, sex, symptomatic events, postdilatation, and dilatation before embolic protection devices (score 3, OR: 10.7, $\mathrm{P}<0.001$; score 4, OR: 11.7, $\mathrm{P}=0.038$ ).

\section{Discussion}

The present study demonstrated that the circumferential degree of carotid calcification, which was easily assessed 

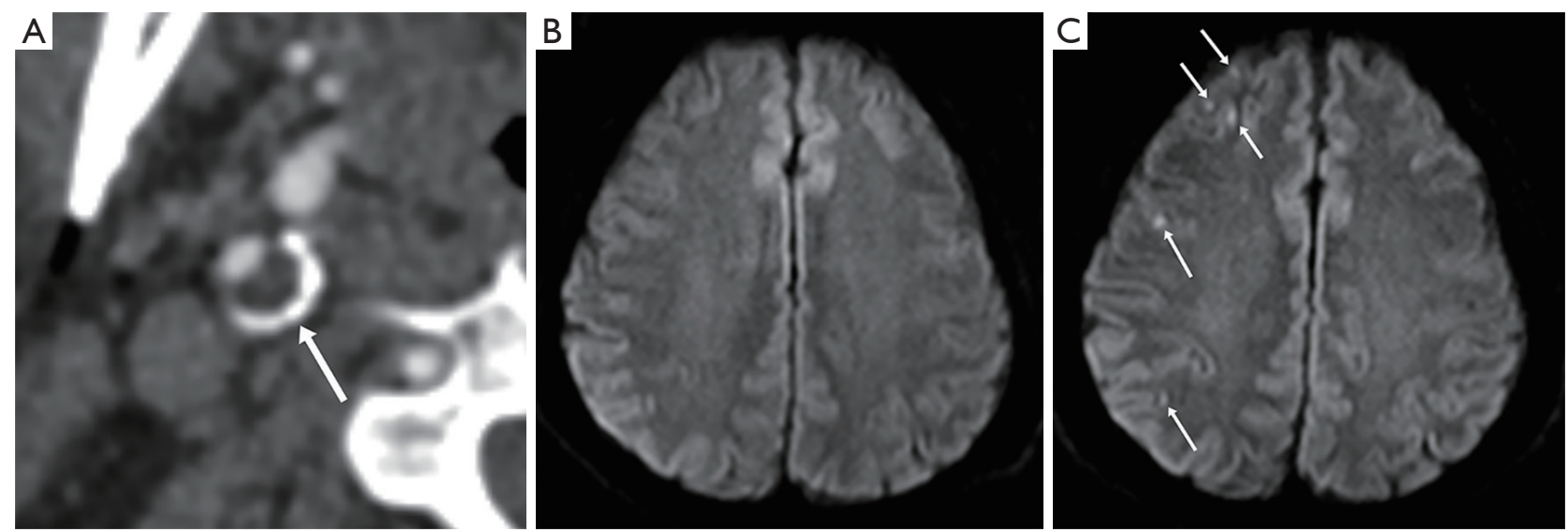

Figure 2 A 60-year-old man with acute cerebral ischemia after carotid artery stenting (CAS). (A) Carotid computed tomography angiography (CTA) shows a right carotid plaque with calcification of $180-270^{\circ}$ of the artery circumference (arrow). (B) Axial diffusion-weighted imaging (DWI) before CAS shows normal findings. (C) Axial DWI after CAS shows multiple ischemic lesions (arrows) in the right cerebral hemisphere.

Table 2 Relationship between plaque calcification and ischemic brain lesions on DWI after CAS

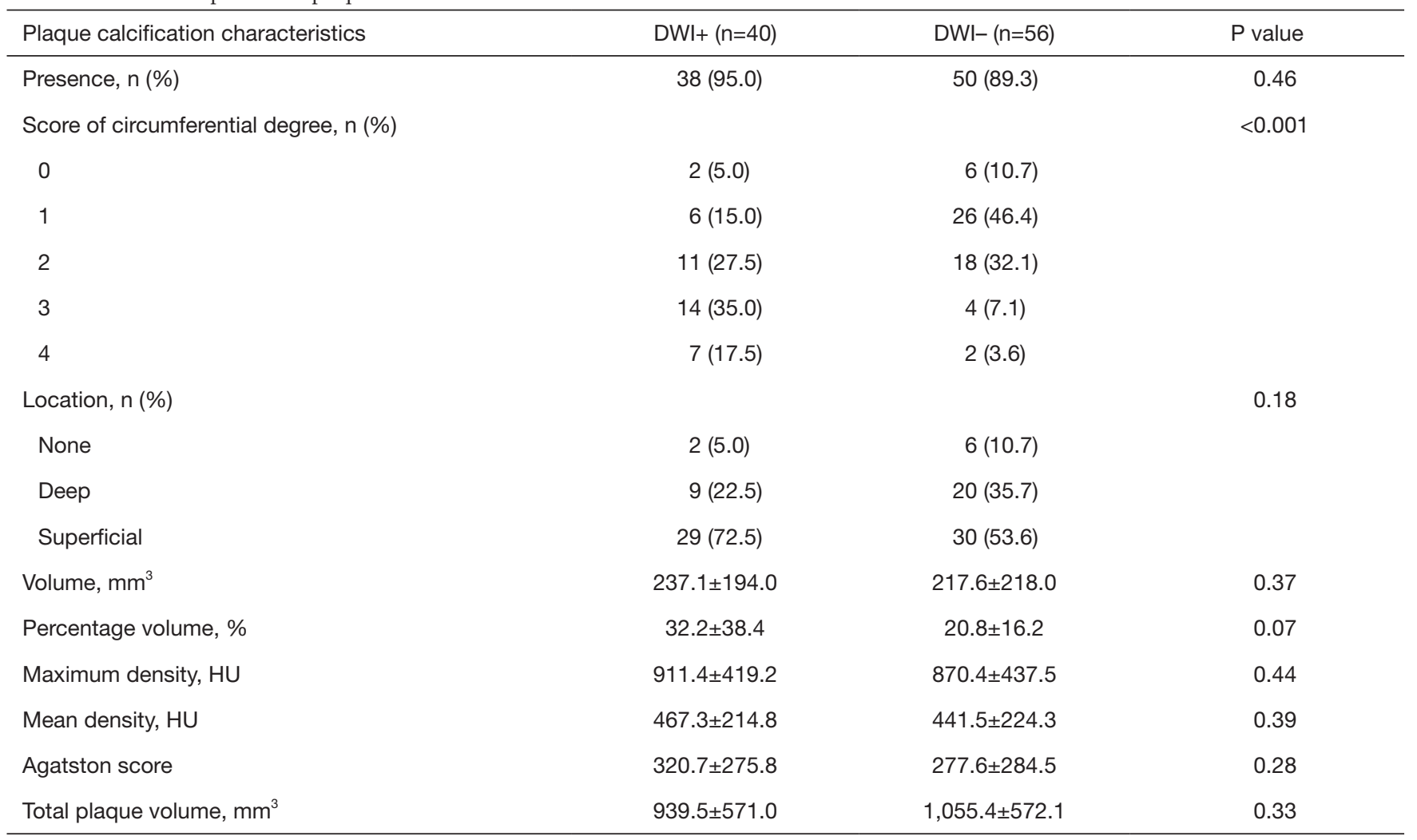

DWI, diffusion-weighted imaging; CAS, carotid artery stenting; HU, Hounsfield unit.

using carotid CT, was associated with new ischemic brain lesions after CAS. Furthermore, the study showed that the circumferential degree of calcification was an independent predictor of new ischemic brain lesions. Preoperative CT evaluation of carotid calcification could help stratify patients with a high risk of cerebral ischemia after CAS. To the best of our knowledge, this is the first report of the association between the circumferential degree of carotid calcification 
Table 3 Univariate and multivariate logistic regression analyses of the association between carotid calcification characteristics and new ischemic brain lesions after CAS

\begin{tabular}{|c|c|c|c|c|}
\hline Variables & \multicolumn{2}{|c|}{ Univariate analysis } & \multicolumn{2}{|c|}{ Multivariate analysis } \\
\hline \multicolumn{5}{|c|}{ Score of circumferential degree } \\
\hline 0 & 1.0 (Reference) & - & 1.0 (Reference) & - \\
\hline 1 & $0.69(0.11-4.31)$ & 0.69 & $0.69(0.10-4.70)$ & 0.70 \\
\hline 3 & $9.7(2.7-35.3)$ & 0.001 & $10.7(2.8-40.7)$ & $<0.001$ \\
\hline 4 & $5.5(1.0-29.2)$ & 0.046 & 11.7 (1.1-119.3) & 0.038 \\
\hline Percentage volume & $1.02(1.00-1.05)$ & 0.07 & $1.02(1.00-1.05)$ & 0.10 \\
\hline
\end{tabular}

CAS, carotid artery stenting; OR, odds ratio.

and new DWI lesions after CAS.

New ischemic brain lesions were commonly found by brain DWI in patients who underwent CAS, with an incidence of $37-50 \%$ (3). In our study, the detection rate of new ischemic brain lesions after CAS was $41.7 \%$, which was consistent with recent literature. The high incidence of ischemic brain lesions may be associated with plaque characteristics. One possible mechanism could be that thrombotic material and atherosclerotic debris are detached from vulnerable plaques during CAS and lead to cerebral embolization $(16,17)$.

Calcification is considered a late process in the atherosclerotic pathophysiology and is common in carotid plaques of moderate and severe stenosis. Besides atherosclerosis, renal dysfunction, metabolic calcium disorder, diabetes, and many other diseases may also lead to calcification of the arterial wall. These etiologies often coexist in elderly people. In our study, 88 of 96 patients (91.7\%) had carotid calcification, while new ischemic brain lesions after CAS were only found in 40 patients (41.7\%). This indicated that the characteristics of the calcification, rather than the presence of calcification, might be related to ischemic brain lesions after CAS. Two previous studies assessed carotid plaque characteristics by virtual histology intravascular ultrasound and found no significant differences in the calcium area between the DWI+ group and the DWI- group $(10,11)$. Another study based on carotid CT also found no correlation between the high-signal DWI lesions and plaque volume of HU >60 (18). Similar results were shown in our study, which supported the notion that the size of the calcification was not significantly associated with cerebral embolism after CAS.

Further analysis by our study found an association between the degree of circumferential carotid calcification and new ischemic brain lesions after carotid stenting. As the stent is pushed open, the vessel wall is stretched to accommodate the stent expansion. However, vessel walls with different plaque components respond differently under mechanical tension, resulting in nonuniform stretching. As the hard calcified plaques are more rigid against deformation, vessel segments with soft plaques on the same circumference have to compensate by overstretching in order to achieve sufficient stent expansion (18). Therefore, when the carotid vessel wall contains considerable circumferential calcification but little soft component, the inserted stent is opened inadequately due to excessive resistance from the rigid vessel wall, with possible consequences being stent recoil and restenosis, which eventually leads to cerebral ischemia (19). Achieving adequate stent expansion requires a significantly greater force, which increases the risk of excessive stretching of the vessel wall. In this situation, the vessel wall could be injured, and the plaque could be fractured, leading to dislodging of both the calcified and noncalcified contents of the plaque $(17,20)$.

It was observed that a large circumferential carotid calcification usually suggests a large volume or percentage volume of calcification. However, the converse is not true: due to the eccentricity of the plaque profile, it cannot be concluded that a large carotid circumference is covered with 
calcification based on a large percentage volume. As a result, even with identical volume of calcification, vessels with different degrees of circumferential calcification can support different expansion strength during stent placement (20). This explains why a similar association between calcification volume and new ischemic brain lesions after CAS is not observed.

Our study had several limitations. First, the number of patients was relatively small, and most patients had carotid stenosis of $>70 \%$. It was unclear whether patients with stenoses of $50-70 \%$ would show the same associations. Therefore, a study involving a larger sample size with a wider range of stenoses is needed. Second, although CT was an ideal imaging examination for the detection of calcification, due to blooming artifacts, overestimation of calcification might have occurred in plaques with heavy calcification (21). However, similar bone window settings were used to identify carotid calcification on the CT images of all the patients, which would mitigate the impact of blooming artifacts on the results (22). Third, we only observed whether new hyperintense lesions existed on postoperative brain DWI; however, the number and size of these lesions could vary, which might affect the patients' rehabilitation. Finally, only calcification was assessed comprehensively in this report, and future studies with a combination of other characteristics of vulnerable plaques in addition to calcification may further improve the reliability of prediction.

\section{Conclusions}

The circumferential degree of carotid calcification was associated with new ischemic brain lesions after CAS. CAS should be avoided if possible for carotid stenosis with large circumferential calcified plaques.

\section{Acknowledgments}

Funding: This work was supported by the National Key Research and Development Program of China (No. 2018YFC1312301).

\section{Footnote}

Conflicts of Interest: All authors have completed the ICMJE uniform disclosure form (available at http://dx.doi. org/10.21037/qims-20-1244). The authors have no conflicts of interest to declare.
Ethical Statement: This study was approved by the ethics committee of our hospital (No. B2020-400R), and individual consent for this retrospective analysis was waived.

Open Access Statement: This is an Open Access article distributed in accordance with the Creative Commons Attribution-NonCommercial-NoDerivs 4.0 International License (CC BY-NC-ND 4.0), which permits the noncommercial replication and distribution of the article with the strict proviso that no changes or edits are made and the original work is properly cited (including links to both the formal publication through the relevant DOI and the license). See: https://creativecommons.org/licenses/by-nc-nd/4.0/.

\section{References}

1. Ji A, Lv P, Dai Y, Bai X, Tang X, Fu C, Lin J. Associations between carotid intraplaque hemorrhage and new ipsilateral ischemic lesions after carotid artery stenting: a quantitative study with conventional multi-contrast MRI. Int J Cardiovasc Imaging 2019;35:1047-54.

2. Kashiwazaki D, Kuwayama N, Akioka N, Noguchi K, Kuroda S. Carotid plaque with expansive arterial remodeling is a risk factor for ischemic complication following carotid artery stenting. Acta Neurochir 2017;159:1299-304.

3. Rots ML, Meershoek AJA, Bonati LH, den Ruijter HM, de Borst GJ. Editor's choice - predictors of new ischaemic brain lesions on diffusion weighted imaging after carotid stenting and endarterectomy: a systematic review. Eur J Vasc Endovasc Surg 2019;58:163-74.

4. Gensicke H, van der Worp HB, Nederkoorn PJ, Macdonald S, Gaines PA, van der Lugt A, Mali WP, Lyrer PA, Peters N, Featherstone RL, de Borst GJ, Engelter ST, Brown MM, Bonati LH. Ischemic brain lesions after carotid artery stenting increase future cerebrovascular risk. J Am Coll Cardiol 2015;65:521-9.

5. Gupta A, Giambrone AE, Gialdini G, Finn C, Delgado D, Gutierrez J, Wright C, Beiser AS, Seshadri S, Pandya A, Kamel H. Silent brain infarction and risk of future stroke: a systematic review and meta-analysis. Stroke 2016;47:719-25.

6. Wei H, Zhang M, Li Y, Zhao X, Canton G, Sun J, Xu D, Zhou Z, Chen S, Ferguson MS, Hatsukami TS, Li R, Yuan C. Evaluation of 3D multi-contrast carotid vessel wall MRI: a comparative study. Quant Imaging Med Surg 2020;10:269-82.

7. Wang Y, Osborne MT, Tung B, Li M, Li Y. Imaging cardiovascular calcification. J Am Heart Assoc 
2018;7:e008564.

8. Pini R, Faggioli G, Fittipaldi S, Pini R, Faggioli G, Fittipaldi S, Vasuri F, Longhi M, Gallitto E, Pasquinelli G, Gargiulo M, Stella A. Relationship between calcification and vulnerability of the carotid plaques. Ann Vasc Surg 2017;44:336-42.

9. White CJ. Carotid artery stenting. J Am Coll Cardiol 2014;64:722-31.

10. Yamada K, Yoshimura S, Kawasaki M, Enomoto Y, Takano K, Asano T, Minatoguchi S, Iwama T. Prediction of silent ischemic lesions after carotid artery stenting using virtual histology intravascular ultrasound. Cerebrovasc Dis 2011;32:106-13.

11. Timaran CH, Rosero EB, Martinez AE, Ilarraza A, Modrall JG, Clagett GP. Atherosclerotic plaque composition assessed by virtual histology intravascular ultrasound and cerebral embolization after carotid stenting. J Vasc Surg 2010;52:1188-94.

12. Baradaran H, Gupta A. Carotid vessel wall imaging on CTA. AJNR Am J Neuroradiol 2020;41:380-6.

13. Barnett HJ, Taylor DW, Eliasziw M, Fox AJ, Ferguson GG, Haynes RB, Rankin RN, Clagett GP, Hachinski VC, Sackett DL, Thorpe KE, Meldrum HE, Spence JD. Benefit of carotid endarterectomy in patients with symptomatic moderate or severe stenosis. North American Symptomatic Carotid Endarterectomy Trial Collaborators. N Engl J Med 1998;339:1415-25.

14. Saba L, Sanfilippo R, Sannia S, Anzidei M, Montisci R, Mallarini G, Suri JS. Association between carotid artery plaque volume, composition, and ulceration: a retrospective assessment with MDCT. AJR Am J Roentgenol 2012;199:151-6.

15. Yamada S, Oshima M, Watanabe Y, Ogata H, Hashimoto $\mathrm{K}$, Miyake H. Intramural location and size of arterial calcification are associated with stenosis at carotid bifurcation. Eur J Radiol 2014;83:957-63.

16. Bonati LH, Jongen LM, Haller S, Flach HZ, Dobson J, Nederkoorn PJ, Macdonald S, Gaines PA, Waaijer A, Stierli P, Jäger HR, Lyrer PA, Kappelle LJ, Wetzel SG,

Cite this article as: Lv P, Ji A, Zhang R, Guo D, Tang X, Lin J. Circumferential degree of carotid calcification is associated with new ischemic brain lesions after carotid artery stenting. Quant Imaging Med Surg 2021;11(6):2669-2676. doi: 10.21037/qims20-1244 van der Lugt A, Mali WP, Brown MM, van der Worp HB, Engelter ST; ICSS-MRI study group. New ischaemic brain lesions on MRI after stenting or endarterectomy for symptomatic carotid stenosis: a substudy of the International Carotid Stenting Study (ICSS). Lancet Neurol 2010;9:353-62. Erratum in: Lancet Neurol 2010;9:345.

17. Matsukawa H, Fujii M, Uemura A, Suzuki K, Yamamoto D, Takahashi O, Niimi Y. Pathology of embolic debris in carotid artery stenting. Acta Neurol Scand 2015;131:197-202.

18. Uchiyama N, Misaki K, Mohri M, Watanabe T, Hirota Y, Nakada M, Hayashi Y, Ueda F, Hamada J. Association between carotid plaque composition assessed by multidetector computed tomography and cerebral embolism after carotid stenting. Neuroradiology 2012;54:487-93.

19. Mazurek A, Partyka L, Trystula M, Jakala J, Proniewska K, Borratynska A, Tomaszewski T, Slezak M, Malinowski KP, Drazkiewicz T, Podolec P, Rosenfiled K, Musialek P. Highly-calcific carotid lesions endovascular management in symptomatic and increased-stroke-risk asymptomatic patients using the CGuard ${ }^{\mathrm{TM}}$ dual-layer carotid stent system: analysis from the PARADIGM study. Catheter Cardiovasc Interv 2019;94:149-56.

20. Barrett HE, Cunnane EM, Kavanagh EG, Walsh MT. On the effect of calcification volume and configuration on the mechanical behaviour of carotid plaque tissue. J Mech Behav Biomed Mater 2016;56:45-56.

21. Mannelli L, MacDonald L, Mancini M, Ferguson M, Shuman WP, Ragucci M, Monti S, Xu D, Yuan C, Mitsumori LM. Dual energy computed tomography quantification of carotid plaques calcification: comparison between monochromatic and polychromatic energies with pathology correlation. Eur Radiol 2015;25:1238-46.

22. Bleeker L, Marquering HA, van den Berg R, Nederkoorn PJ, Majoie CB. Semi-automatic quantitative measurements of intracranial internal carotid artery stenosis and calcification using CT angiography. Neuroradiology 2012;54:919-27. 